\title{
Evaluation of serum CEA and CA19-9 levels as prognostic factors in patients with gastric cancer
}

\author{
Mitsugu Kochi, Masashi Fuji, Noriaki Kanamori, Teruo Kaiga, Tetsuya Kawakami, Kazuo Aizaki, \\ Mitsuko Kasahara, Fumiro Mochizuki, Yuichi Kasakura, and Motoo Yamagata \\ Third Department of Surgery, Nihon University School of Medicine, 30-1 Ohyaguchi Kamimachi, Itabashi-ku, Tokyo 173-8610, Japan
}

\begin{abstract}
Background. This clinicopathological study evaluated the utility of serum carcinoembryonic antigen (CEA) and carbohydrate antigen (CA) 19-9 as predictors of locoregional recurrence and long-term disease-free survival in patients with gastric cancer.

Methods. During the period January 1989 to December 1994, 485 patients with primary gastric cancer were evaluated. Gastrectomies were performed in 434 patients. Prognostic factors were analyzed by the Kaplan-Meier method and multivariate analysis, using Cox regression.

Results. Elevated serum CEA and CA19-9 levels were observed in 92 of the 485 patients $(19.0 \%)$, and in 95 of the 435 patients $(21.8 \%)$, respectively, and both markers were elevated in 29 of these 435 patients $(6.7 \%)$. Elevated serum CEA and CA19-9 levels correlated well with lymph node metastasis, lymphatic invasion, vessel invasion, stage grouping, depth of invasion, and curability. Patients with elevated serum CEA levels were at significantly higher risk of having all recurrence factors than were those with normal serum CEA levels. Patients with elevated serum CA19-9 levels were at significantly higher risk of having peritoneal metastases and distant metastases than were those with normal serum CA199 levels. A significant difference in the cumulative survival curves of patients was demonstrated between those with elevated and those with normal serum CEA or CA19-9 levels, even for patients at the same disease stage (stage III). Patients with elevated levels of both markers had a significantly worse prognosis than patients in whom the levels of both markers were normal. In patients who underwent gastrectomy, elevated serum CEA levels either preoperatively or within 3 weeks after gastrectomy were associated with significantly worse prognosis than were normal levels. When the cutoff level of serum CEA was increased to $10 \mathrm{ng} / \mathrm{ml}$, serum CEA, age, lymph node metastasis, and surgical stage grouping were
\end{abstract}

Offprint requests to: $\mathrm{M}$. Kochi

Received: June 20, 2000 / Accepted: November 14, 2000 selected as independent prognostic factors by multivariate analysis of 14 prognostic factors, using Cox regression.

Conclusion. Serum CEA and CA19-9 levels provide additional prognostic information in patients with primary gastric cancer. In particular, an elevated serum CEA level provides additional prognostic information and is a useful indicator of curability in patients who undergo gastrectomy. Serum CEA level is an independent prognostic factor in patients with primary gastric cancer.

Key words Tumor marker · CEA · CA19-9 - Gastric cancer · Prognosis

\section{Introduction}

In spite of recent improvements in early detection, progress in surgical techniques, and the development of adjuvant chemotherapeutic regimens, the survival of patients with gastric cancer still needs to be improved [1]. Although morbidity and mortality have decreased significantly in the past 40 years, the overall 5-year survival in Japanese patients with gastric cancer remains less than $70 \%$ [2]. Distant metastasis, depth of invasion, and lymph node metastasis are well known to be strong prognostic factors [3-6], and, in addition, serum tumor marker levels are considered to be important. Various tumor markers have been described since Gold and Freedman [7] reported the discovery of carcinoembryonic antigen (CEA) in 1965. Serum CEA levels have been studied in an effort to identify those patients who may be at increased risk of recurrence despite curative resection and who are therefore appropriate candidates for adjuvant therapy. In colon cancer, Holyoke et al. [8] reported that when the CEA cutoff value was set at $2.5 \mathrm{ng} / \mathrm{dl}$, there were more recurrences among patients whose preoperative CEA values were above this level. Wanebo et al. [9] reported that when the cutoff value was set at $5 \mathrm{ng} / \mathrm{dl}$, there were significantly more recur- 
rences in patients with Dukes $\mathrm{B}(P<0.02)$ and Dukes $\mathrm{C}$ $(P<0.001)$, suggesting that CEA-positive patients had significantly poorer prognoses.

Another tumor marker, carbohydrate antigen (CA) 19-9 [10], a ligand of E-selectin [11], is a molecule that may play a role in the adhesion of cancer cells to endothelial cells, resulting in hematogenic metastasis. A significant difference has been observed between the prognosis of stage II-III pancreas cancer patients with and without elevated CA19-9 levels [12]. Pronounced expression of CA19-9 has also been observed by immunostaining of gastric cancer tissue [13], making it another of the tumor markers that are frequently evaluated in gastric cancer patients in Japan.

Although serum CEA may be useful in the followup of colon cancer to identify tumor recurrence, and CA19-9 may be useful in the follow-up of pancreas cancer, it is, at present, unclear what role tumor markers may have in predicting or identifying those patients with gastric cancer who will develop recurrence after curative resection. Therefore, in this clinicopathological study, we evaluated whether serum CEA and CA19-9 levels predicted locoregional recurrence and long-term disease-free survival in patients with gastric cancer.

\section{Patients and methods}

Between January 1989 and December 1994, 485 patients with primary gastric cancer were referred for evaluation. Gastrectomies were performed in 434 patients (resectability rate $89.5 \%$ ). Of the 434 patients, total gastrectomy was performed in $129(29.7 \%)$, subtotal gastrectomy in 287 (66.1\%), and wedge resection in 18 patients $(4.1 \%)$. All patients had histologically proven adenocarcinoma of the stomach. We selected the following 12 prognostic factors for evaluation: age, sex, main tumor location, tumor size, gross type, clinical stage, histological type, depth of invasion, lymph node metastasis, lymphatic and venous invasion, and curability. For main tumor location, the stomach was divided into upper, middle, and lower positions; histological type was assessed as tub1, tub2, por1, por2, and sig types; depth of invasion ( $\mathrm{t} 1, \mathrm{t} 2, \mathrm{t} 3, \mathrm{t} 4)$, lymphatic and venous invasion, and curability $(\mathrm{A}, \mathrm{B}, \mathrm{C})$ were classified according to the Japanese classification of gastric cancer (JCGC) [16]. Serum CEA and CA19-9 levels were analyzed in relation to these prognostic factors by multivariate analysis. The preoperative CEA and CA19-9 levels (within 1 month prior to gastrectomy) and the postoperative CEA and CA19-9 levels (within 3 weeks after gastrectomy) were determined in the clinical laboratory. Serum levels of CEA were determined with a commercial enzyme immunoassay kit (Fujirebio; Tokyo, Japan). Serum levels of CA19-9 were also evalu- ated with a commercial enzyme immunoassay kit (Fujirebio). The cutoff value for CEA was $5 \mathrm{ng} / \mathrm{ml}$ and that for CA19-9 was $37 \mathrm{U} / \mathrm{ml}$.

Cumulative survival rates were calculated by the Kaplan-Meier method, and statistical significance was evaluated by the log-rank test. The Survival Tools for StatView program (Abacus Concepts, Barkeley, CA, USA) was used for simultaneous multivariate adjustment of all covariates by Cox regression analysis. Forward stepwise regression analysis (likelihood-ratio statistic) was performed to select good predictors of survival. The relative risk of death was compared by using the exponential coefficient $[\exp (\beta)]$. Statistical significance was assumed for a $P$ value of less than 0.05 .

\section{Results}

\section{Clinicopathological and surgical features}

The patients' clinicopathological and surgical features are shown in Table 1 . Their median age was 61.9 years (range, 20-90 years). The ratio of males to females was $3: 1$. Serum levels of CEA were elevated in 92 of the 485 patients $(19.0 \%)$, CA19-9 levels were elevated in 95 of the 435 patients $(21.8 \%)$, and both markers were elevated in 29 of these 435 patients $(6.7 \%)$. There were no significant differences according to sex between elevated and normal levels for either serum CEA or CA19-9 levels. The proportions of patients with elevated serum CEA and CA19-9 levels were significantly higher in those at stage IV of the JCGC surgical stage grouping than the proportions in patients at other stages (CEA, 53.9\%; CA19-9, 51.6\%). At stage III and below, the positivity rates for elevated CEA and CA19-9 levels were below 30\%. Lymph node metastasis, lymphatic invasion, vessel invasion, stage grouping, depth of invasion, and curability were significantly different between the patients with elevated and those with normal serum levels of either CEA or CA19-9.

The proportions of patients with elevated and those with normal levels of either serum CEA or CA19-9 were compared according to JCGC histological type, but there were no significant differences. However, the proportion of patients with elevated serum CEA levels tended to be higher in those with tub2, and lower in those with tub1 and sig. Elevated serum CA19-9 levels tended to be more frequent in patients with tub2, and lower in those with sig.

\section{Recurrence type}

Recurrence type was compared in patients with elevated and those with normal serum levels of either 
Table 1. Clinicopathological characteristics

\begin{tabular}{|c|c|c|c|c|c|c|c|}
\hline & & \multicolumn{2}{|c|}{ Serum CEA $(\mathrm{n}=485)$} & \multirow[b]{2}{*}{$P$} & \multicolumn{2}{|c|}{ Serum CA19-9 $(n=435)$} & \multirow[b]{2}{*}{$P$} \\
\hline & & Normal & Elevated & & Normal & Elevated & \\
\hline Sex & $\begin{array}{l}\text { Male } \\
\text { Female }\end{array}$ & $\begin{array}{l}286(72.8) \\
107(27.2)\end{array}$ & $\begin{array}{l}69(75.0) \\
23(25.0)\end{array}$ & NS & $\begin{array}{r}253(74.4) \\
87(25.6)\end{array}$ & $\begin{array}{l}64(67.4) \\
31(32.6)\end{array}$ & NS \\
\hline Age (years) & $\begin{array}{l}\text { Median } \\
\text { Range }\end{array}$ & $\begin{array}{l}61.2 \\
27-90\end{array}$ & $\begin{array}{l}61.9 \\
20-90\end{array}$ & NS & $\begin{array}{l}60.9 \\
20-90\end{array}$ & $\begin{array}{l}66.4 \\
34-90\end{array}$ & NS \\
\hline Location & $\begin{array}{l}\text { Upper } \\
\text { Middle } \\
\text { Lower }\end{array}$ & $\begin{array}{r}73(18.9) \\
173(44.7) \\
141(36.4)\end{array}$ & $\begin{array}{l}22(25.3) \\
29(33.3) \\
36(41.4)\end{array}$ & NS & $\begin{array}{r}68(20.2) \\
143(42.4) \\
126(37.4)\end{array}$ & $\begin{array}{l}20(22.2) \\
38(41.8) \\
33(36.3)\end{array}$ & NS \\
\hline Tumor size (mm) & & 48.3 & 56.4 & NS & 42.7 & 72.7 & NS \\
\hline Gross type & $\begin{array}{l}\text { Ulcerated } \\
\text { Polypoid } \\
\text { Diffuse } \\
\text { Nonclass }\end{array}$ & $\begin{array}{r}256(67.4) \\
54(14.2) \\
42(11.1) \\
28(7.4)\end{array}$ & $\begin{aligned} 67 & (78.8) \\
2 & (2.3) \\
11 & (12.9) \\
5 & (5.9)\end{aligned}$ & NS & $\begin{array}{c}233(70.6) \\
38(11.5) \\
36(10.9) \\
23(7.0)\end{array}$ & $\begin{array}{c}54(61.4) \\
10(11.4) \\
17(19.3) \\
7(8.0)\end{array}$ & \\
\hline Stage & $\begin{array}{l}\text { I } \\
\text { II } \\
\text { III } \\
\text { IV }\end{array}$ & $\begin{array}{r}176(46.3) \\
55(14.2) \\
78(20.5) \\
71(18.7)\end{array}$ & $\begin{array}{l}13(14.6) \\
12(13.5) \\
16(18.0) \\
48(53.9)\end{array}$ & $P<0.001$ & $\begin{array}{r}139(41.7) \\
38(11.5) \\
73(21.9) \\
65(19.5)\end{array}$ & $\begin{array}{l}24(25.3) \\
10(10.5) \\
12(12.6) \\
49(51.6)\end{array}$ & $P<0.001$ \\
\hline Histologic type & $\begin{array}{l}\text { tub1 } \\
\text { tub2 } \\
\text { por1 } \\
\text { por2 } \\
\text { sig }\end{array}$ & $\begin{aligned} 54 & (14.8) \\
103 & (28.3) \\
8 & (2.2) \\
161 & (44.2) \\
38 & (10.4)\end{aligned}$ & $\begin{aligned} 7 & (8.9) \\
31 & (39.2) \\
3 & (3.8) \\
34 & (43.0) \\
4 & (5.1)\end{aligned}$ & NS & $\begin{array}{r}43(13.6) \\
94(29.7) \\
8(2.5) \\
133(42.1) \\
38(12.0)\end{array}$ & $\begin{aligned} 10 & (12.8) \\
27 & (34.6) \\
3 & (3.8) \\
35 & (44.9) \\
3 & (3.8)\end{aligned}$ & NS \\
\hline Depth of invasion & $\begin{array}{l}\mathrm{t} 1 \\
\mathrm{t} 2 \\
\mathrm{t} 3 \\
\mathrm{t} 4\end{array}$ & $\begin{array}{r}165(47.3) \\
105(30.1) \\
74(21.2) \\
5(1.4)\end{array}$ & $\begin{array}{c}16(24.3) \\
31(46.9) \\
16(24.2) \\
3(4.5)\end{array}$ & $P<0.005$ & $\begin{array}{c}132(43.6) \\
111(36.6) \\
58(19.1) \\
2(0.7)\end{array}$ & $\begin{array}{c}22(32.4) \\
17(25.0) \\
26(38.2) \\
3(3.8)\end{array}$ & $P<0.005$ \\
\hline Lymph node meta & $\begin{array}{l}\text { n0 } \\
\text { n1 } \\
\text { n2 } \\
\text { n3 }\end{array}$ & $\begin{array}{r}215(63.6) \\
70(20.7) \\
42(12.4) \\
11(3.3)\end{array}$ & $\begin{array}{r}20(33.9) \\
20(33.9) \\
10(16.9) \\
9(15.3)\end{array}$ & $P<0.0001$ & $\begin{aligned} 181 & (61.1) \\
67 & (22.6) \\
41 & (13.9) \\
7 & (2.4)\end{aligned}$ & $\begin{aligned} 29 & (50.0) \\
12 & (20.7) \\
5 & (8.6) \\
12 & (20.7)\end{aligned}$ & $P<0.005$ \\
\hline Lymphatic invasion & $\begin{array}{l}\text { ly0 } \\
\text { ly1 } \\
\text { ly2 } \\
\text { ly3 }\end{array}$ & $\begin{array}{r}135(38.8) \\
89(25.6) \\
78(22.4) \\
46(13.2)\end{array}$ & $\begin{array}{l}12(18.2) \\
14(21.2) \\
23(34.8) \\
17(25.8)\end{array}$ & $P<0.005$ & $\begin{array}{r}113(37.2) \\
78(25.7) \\
77(25.3) \\
36(11.8)\end{array}$ & $\begin{array}{l}18(26.9) \\
15(22.4) \\
10(14.9) \\
24(35.8)\end{array}$ & $P<0.0001$ \\
\hline Vessel invasion & $\begin{array}{l}\text { v0 } \\
\text { v1 } \\
\text { v2 } \\
\text { v3 }\end{array}$ & $\begin{array}{r}259(74.6) \\
87(19.3) \\
22(5.2) \\
6(0.9)\end{array}$ & $\begin{array}{c}39(59.1) \\
20(30.3) \\
4(6.1) \\
3(4.5)\end{array}$ & $P<0.05$ & $\begin{array}{r}220(72.6) \\
64(21.1) \\
17(5.6) \\
2(0.7)\end{array}$ & $\begin{array}{c}44(65.7) \\
16(23.9) \\
3(4.4) \\
4(6.0)\end{array}$ & $P<0.05$ \\
\hline Curability & $\begin{array}{l}\mathrm{A} \\
\mathrm{B} \\
\mathrm{C}\end{array}$ & $\begin{array}{r}184(46.8) \\
126(32.1) \\
83(21.1)\end{array}$ & $\begin{array}{l}16(22.5) \\
27(38.0) \\
28(39.4)\end{array}$ & $P<0.0005$ & $\begin{array}{r}155(50.2) \\
115(37.2) \\
39(12.6)\end{array}$ & $\begin{array}{ll}21 & (29.2) \\
23 & (31.9) \\
28 & (38.9)\end{array}$ & $P<0.0001$ \\
\hline
\end{tabular}

Numbers in parentheses are percentages CEA, Carcinoembryonic antigen; CA19-9, carbohydrate antigen 19-9; NS, not significant

CEA or CA19-9, using four recurrence factors: lymph node metastases, peritoneal metastases, liver metastases, and distant metastases (Table 2). Patients with elevated serum CEA levels were at significantly higher risk of having all recurrence factors than were those with normal serum CEA levels. All factors were analyzed by forward stepwise selection. Liver metastases, distant metastases, and lymph node metastases were selected as independent risk factors, and liver metastasis was the highest risk factor for elevated serum CEA levels. Patients with elevated serum CA19-9 levels were at significantly higher risk of having peritoneal metastases and distant metastases than were those with normal serum CA19-9 levels. Distant metastases and peritoneal metastases were selected as independent risk factors, and distant metastasis was the highest risk factor for elevated serum CA19-9 levels.

\section{Survival rate}

Cumulative survival was compared in patients with primary gastric cancer who had elevated and those who 
Table 2. Elevated and normal serum levels of either CEA or CA19-9 according to four recurrence factors

\begin{tabular}{|c|c|c|c|c|c|c|c|}
\hline & & \multicolumn{2}{|c|}{ Serum CEA } & & \multicolumn{2}{|c|}{ Serum 19-9 } & \\
\hline & & Normal & Elevated & & Normal & Elevated & \\
\hline \multicolumn{8}{|c|}{ Recurrence factor } \\
\hline & Positive & $15(3.8)$ & $20(22.0)$ & $P<0.0001$ & $23(6.8)$ & $7(7.5)$ & NS \\
\hline & Negative & $376(96.2)$ & $71(78.0)$ & & $315(93.2)$ & $86(92.5)$ & \\
\hline & Positive & $131(33.3)$ & $71(77.2)$ & $P<0.0001$ & $217(56.5)$ & $57(62.0)$ & NS \\
\hline & Negative & $262(66.6)$ & $21(22.8)$ & & $167(43.5)$ & $35(38.0)$ & \\
\hline \multirow[t]{2}{*}{$\mathrm{P}$} & Positive & $35(8.9)$ & $18(19.8)$ & $P<0.005$ & $32(9.4)$ & $19(20.4)$ & $P<0.005$ \\
\hline & Negative & $357(91.1)$ & $73(80.2)$ & & $310(90.6)$ & $74(79.6)$ & \\
\hline & Positive & $9(2.3)$ & $10(11.1)$ & $P<0.0001$ & $7(2.0)$ & $9(9.7)$ & $P<0.0005$ \\
\hline & Negative & $382(97.7)$ & $80(88.9)$ & & $335(98.0)$ & $84(90.3)$ & \\
\hline
\end{tabular}

H, Liver metastasis; N, lymph node metastasis; P, peritoneal metastasis; M, distant metastasis
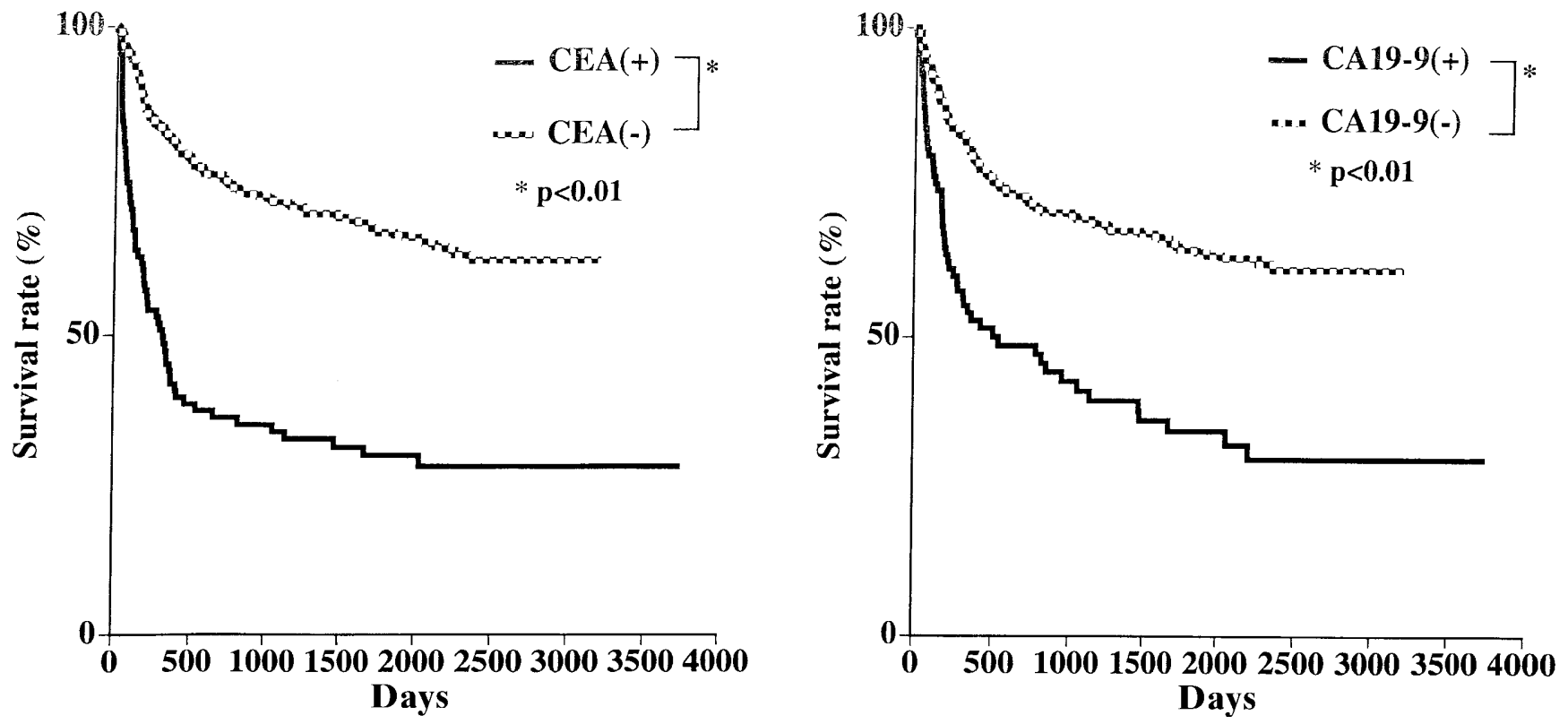

Fig. 1. Comparison of cumulative survival curves according to serum carcinoembryonic antigen $(C E A)$ level and carbohydrate antigen $(C A)$ 19-9 level. Patients with elevated serum CEA or elevated CA 19-9 levels had a significantly worse prognosis than patients with normal levels of either marker $(P<0.01)$

had normal serum levels of CEA or CA19-9. Patients with elevated serum CEA or CA19-9 levels had a significantly worse prognosis than patients with normal levels of either tumor marker (Fig. 1. $P<0.01$ for both markers). To evaluate combination assays of serum CEA and CA19-9 levels, cumulative survivorship was compared in four groups (both markers elevated, both normal elevated CEA and normal CA19-9, and elevated CA19-9 and normal CEA) (Fig. 2). Patients in whom serum levels of both CEA and CA19-9 were elevated had significantly worse prognoses than patients in whom the levels of both markers were normal $(P<$ $0.0001)$. No significant difference was demonstrated be- tween patients with an elevated serum level of CEA and a normal serum level of CA19-9, and those with an elevated serum level of CA19-9 and a normal serum level of CEA.

In this analysis, however, bias may have been introduced by considering the different stages of cancer together. In order to eliminate such bias, cumulative survival rates were compared by surgical stage. Comparison of cumulative survival curves according to the JCGC surgical stage grouping demonstrated that patients at stage III with elevated serum CEA or CA19-9 levels had a significantly worse prognosis than those with normal levels of either tumor marker (Fig. 3; $P<$ 


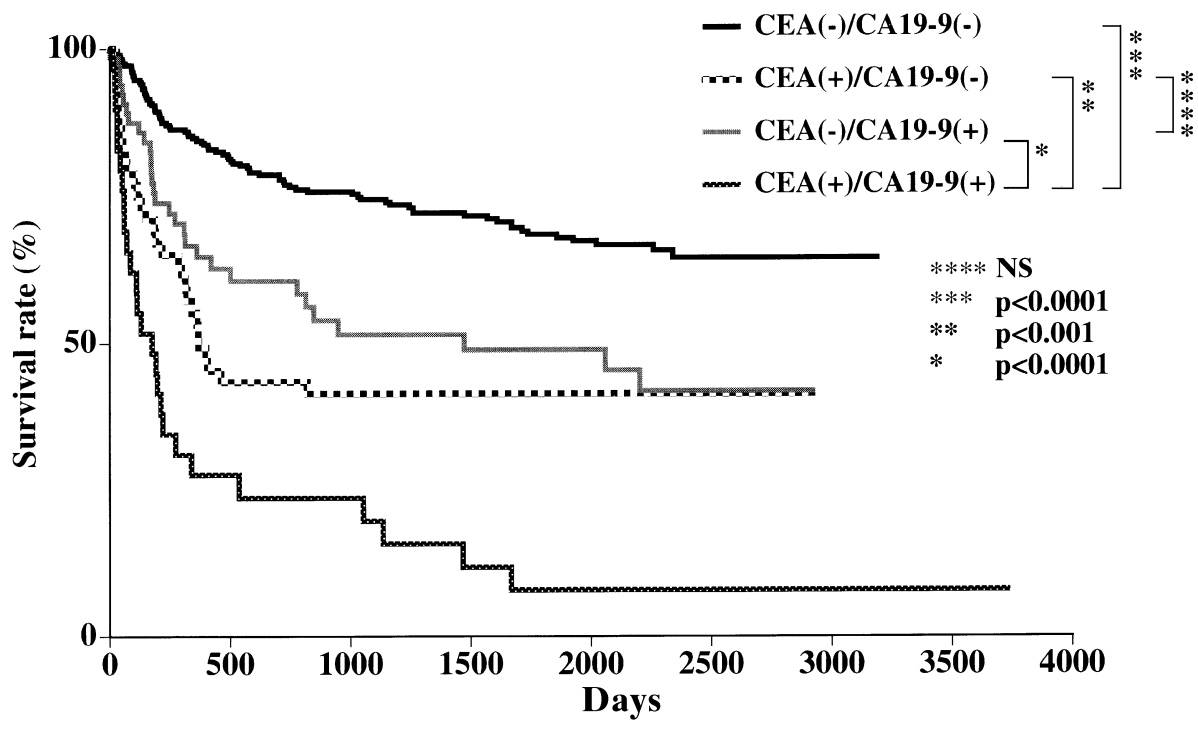

Fig. 2. Comparison of cumulative survival curves according to four combinations of serum CEA and CA 19-9 levels. The differences between all groups, except for that between CEA $(+) / C A 19-9(-)$ and $\operatorname{CEA}(-)$ and CA19-9 $(+)$ were significant $(P<0.001)$
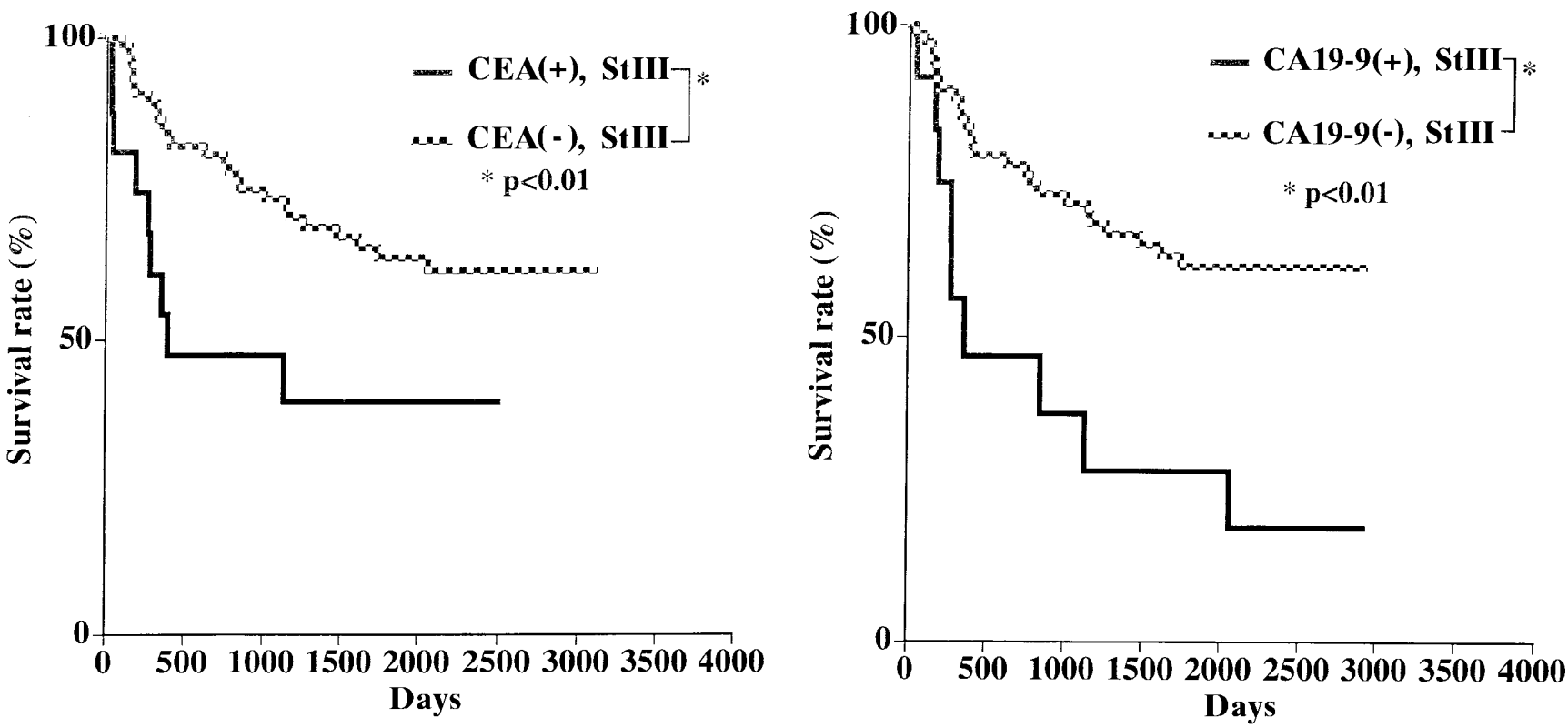

Fig. 3. Comparison of cumulative survival curves in patients classified according to the Japanese classification of gastric cancer (JCGC) surgical stage grouping. Patients at stage III with elevated serum CEA and CA 19-9 levels had a significantly worse prognosis than did patients with normal CEA and CA19-9 levels $(P<0.01)$

0.01 for both markers). On the other hand for stages I, II, and IV, there were no significant differences between patients in whom the serum level of either CEA or CA19-9 was elevated and that of the either marker was normal. Cumulative survival was also compared in patients with elevated and those with normal serum levels of either CEA or CA19-9 who were classified according to the curative potential of gastrectomy by the JCGC criteria. Patients with elevated serum CEA levels had significantly worse prognoses $(P<0.05)$ than did patients with normal levels (Fig. 4). However, no significant difference was demonstrated between patients with elevated and those with normal serum CA19-9 levels.

Finally, cumulative survival was studied for patients who showed elevated serum CEA or CA19-9 levels before gastrectomy, in terms of their levels within 3 weeks after gastrectomy. There was a significant difference in survival $(P<0.01)$ between patients whose serum CEA levels remained elevated after gastrectomy 


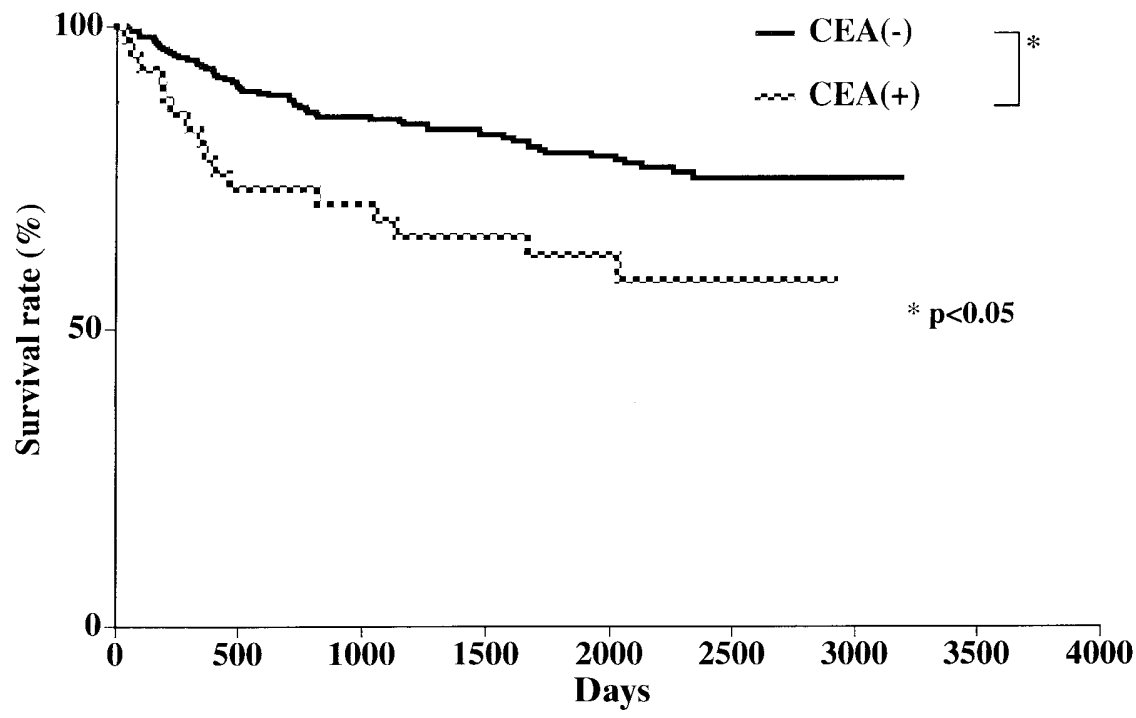

Fig. 4. Cumulative survival was compared in patients with elevated and those with normal serum CEA levels who were classified according to curative potential of gastrectomy by the JCGC criteria. Patients with elevated serum CEA levels had a significantly worse prognosis than did patients with normal CEA levels $(P<$ $0.05)$

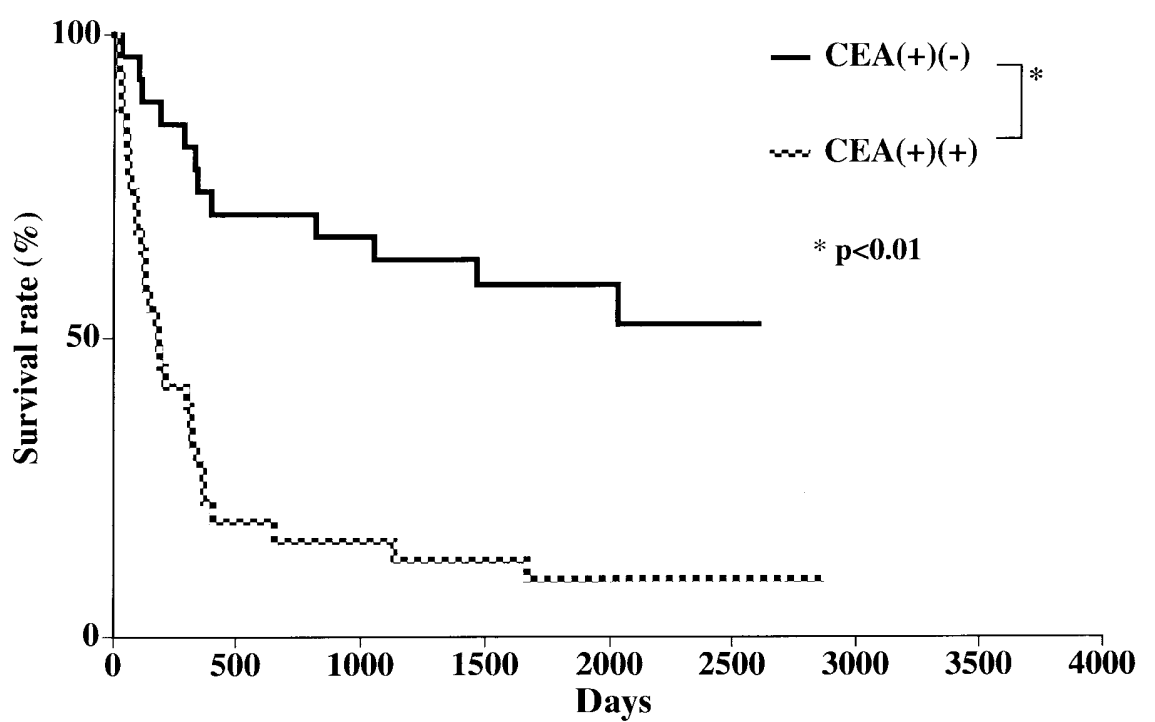

Fig. 5. Comparison of cumulative survival curves of patients with elevated serum CEA levels before gastrectomy according to serum CEA level after resection. A significant difference $(P<0.01)$ was demonstrated between patients whose elevated serum CEA levels became normal after gastrectomy and those whose CEA levels remained elevated and those whose serum CEA levels became normal (Fig. 5). However, no such significant difference was demonstrated in patients with elevated serum CA19-9 levels.

\section{Comparison of hazard ratios}

The 14 prognostic factors and either the CEA or CA199 level were analyzed by multivariate analysis, using Cox regression (Table 3). In all analyses, depth of invasion showed the highest $[\exp (\beta)$, value and serum CEA levels showed higher $[\exp (\beta)$ values than serum CA199. The serum CA19-9 level was not shown to be a highly significant prognostic factor. Elevation of both markers was not shown to be a significantly high prognostic factor $(P<0.1)$. All factors were analyzed by forward stepwise selection with Cox regression. When the cutoff level of serum CEA was increased to $10 \mathrm{ng} / \mathrm{ml}$, serum CEA, age, lymph node metastasis, and surgical stage grouping were selected as independent prognostic factors (Table 4).

\section{Discussion}

In this clinicopathological study, we evaluated the utility of serum CEA and CA19-9 as predictors of locoregional recurrence and long-term disease-free survival in patients with gastric cancer. Of the 485 patients examined, $73.2 \%$ were men and $26.8 \%$ were women. The ratio of males to females was $3: 1$, and there were no significant sex differences between patients with el- 
Table 3. Multivariate analysis of prognostic factors by Cox regression

\begin{tabular}{|c|c|c|c|c|c|c|}
\hline & Category & $\beta$ & SE & Wald & $P$ value & $\operatorname{Exp}(\beta)$ \\
\hline $\mathrm{CEA}(+)$ & $\begin{array}{l}\text { Normal } \\
\text { Elevated }\end{array}$ & $\begin{array}{l}0 \\
0.663\end{array}$ & 0.33 & 2.012 & 0.0442 & 1.941 \\
\hline CA19-9(+) & $\begin{array}{l}\text { Elevated } \\
\text { Normal }\end{array}$ & $\begin{array}{l}0 \\
0.546\end{array}$ & 0.397 & 1.376 & 0.1689 & 1.726 \\
\hline $\operatorname{CEA}(+), \operatorname{CA} 19-9(+)$ & $\begin{array}{l}\text { Elevated } \\
\text { Normal }\end{array}$ & $\begin{array}{l}0 \\
1.023\end{array}$ & 0.63 & 1.624 & 0.1044 & 2.781 \\
\hline Age (years) & & 0.25 & 0.011 & 2.248 & 0.0246 & 1.026 \\
\hline Sex & $\begin{array}{l}\text { Female } \\
\text { Male }\end{array}$ & $\begin{array}{l}0 \\
0.005\end{array}$ & 0.277 & 0.017 & 0.9862 & 1.005 \\
\hline Location & $\begin{array}{l}\text { Middle } \\
\text { Lower } \\
\text { Upper }\end{array}$ & $\begin{array}{l}0.556 \\
0.015\end{array}$ & $\begin{array}{l}0.293 \\
0.308\end{array}$ & $\begin{array}{l}1.902 \\
0.05\end{array}$ & $\begin{array}{l}0.1082 \\
0.0572 \\
0.96\end{array}$ & $\begin{array}{l}1.744 \\
1.016\end{array}$ \\
\hline Gross type & $\begin{array}{l}\text { Ulcerated } \\
\text { Polypoid } \\
\text { Diffuse } \\
\text { Nonclass }\end{array}$ & $\begin{array}{l}0.761 \\
0.67 \\
0.377\end{array}$ & $\begin{array}{l}0.415 \\
0.36 \\
0.458\end{array}$ & $\begin{array}{l}1.835 \\
1.863 \\
0.824\end{array}$ & $\begin{array}{l}0.1229 \\
0.0666 \\
0.0624 \\
0.4099\end{array}$ & $\begin{array}{l}2.141 \\
1.954 \\
1.459\end{array}$ \\
\hline Stage & $\begin{array}{l}\text { III } \\
\text { I } \\
\text { II } \\
\text { IV }\end{array}$ & $\begin{array}{l}0.089 \\
0.273 \\
0.757\end{array}$ & $\begin{array}{l}0.629 \\
0.37 \\
0.379\end{array}$ & $\begin{array}{r}0.141 \\
0.737 \\
21.996\end{array}$ & $\begin{array}{l}0.2407 \\
0.8881 \\
0.4613 \\
0.046\end{array}$ & $\begin{array}{l}1.093 \\
1.313 \\
2.132\end{array}$ \\
\hline Depth of invasion & $\begin{array}{l}\text { t1 } \\
\text { t2 } \\
\text { t3 } \\
\text { t4 }\end{array}$ & $\begin{array}{l}3.178 \\
1.682 \\
0.811\end{array}$ & $\begin{array}{l}1.272 \\
0.531 \\
0.486\end{array}$ & $\begin{array}{r}2.499 \\
23.166 \\
1.667\end{array}$ & $\begin{array}{l}0.0019 \\
0.0124 \\
0.0015 \\
0.0955\end{array}$ & $\begin{array}{c}24.007 \\
5.375 \\
2.25\end{array}$ \\
\hline Histological type & $\begin{array}{l}\text { pol1 } \\
\text { pol2 } \\
\text { tub1 } \\
\text { tub2 } \\
\text { Sig }\end{array}$ & $\begin{array}{l}0.547 \\
0.795 \\
1.086 \\
0.329\end{array}$ & $\begin{array}{l}0.777 \\
0.861 \\
0.771 \\
0.97\end{array}$ & $\begin{array}{l}0.704 \\
0.923 \\
1.408 \\
0.339\end{array}$ & $\begin{array}{l}0.2513 \\
0.4816 \\
0.356 \\
0.159 \\
0.7348\end{array}$ & $\begin{array}{r}11.728 \\
2.213 \\
2.962 \\
1.389\end{array}$ \\
\hline Lymph node meta & $\begin{array}{l}\text { n0 } \\
\text { n1 } \\
\text { n2 } \\
\text { n3 }\end{array}$ & $\begin{array}{l}0.565 \\
0.466 \\
1.009\end{array}$ & $\begin{array}{l}0.34 \\
0.39 \\
0.527\end{array}$ & $\begin{array}{l}1.663 \\
1.192 \\
1.914\end{array}$ & $\begin{array}{l}0.2463 \\
0.0963 \\
0.2331 \\
0.0556\end{array}$ & $\begin{array}{l}1.76 \\
1.593 \\
2.743\end{array}$ \\
\hline Lymphatic invasion & $\begin{array}{l}\text { ly2 } \\
\text { ly0 } \\
\text { ly1 } \\
\text { ly3 }\end{array}$ & $\begin{array}{l}0.225 \\
0.62 \\
0.821\end{array}$ & $\begin{array}{l}0.517 \\
0.356 \\
0.319\end{array}$ & $\begin{array}{l}0.435 \\
1.745 \\
2.578\end{array}$ & $\begin{array}{l}0.0619 \\
0.6639 \\
0.081 \\
0.01\end{array}$ & $\begin{array}{l}1.252 \\
1.86 \\
2.274\end{array}$ \\
\hline Venous invasion & $\begin{array}{l}\text { v0 } \\
\text { v1 } \\
\text { v2 } \\
\text { v3 }\end{array}$ & $\begin{array}{l}0.062 \\
0.002 \\
2.499\end{array}$ & $\begin{array}{l}0.326 \\
0.467 \\
0.612\end{array}$ & $\begin{array}{l}0.189 \\
0.004 \\
4.084\end{array}$ & $\begin{array}{r}0.0003 \\
0.8504 \\
0.9997 \\
<0.0001\end{array}$ & $\begin{array}{r}1.063 \\
1.002 \\
12.166\end{array}$ \\
\hline Curability & $\begin{array}{l}\mathrm{A} \\
\mathrm{B} \\
\mathrm{C}\end{array}$ & $\begin{array}{l}0.567 \\
1.013\end{array}$ & $\begin{array}{l}0.405 \\
0.567\end{array}$ & $\begin{array}{l}1.399 \\
1.786\end{array}$ & $\begin{array}{l}0.2 \\
0.1617 \\
0.074\end{array}$ & $\begin{array}{l}1.763 \\
2.754\end{array}$ \\
\hline
\end{tabular}

evated and those with normal serum levels for either CEA or CA19-9. According to a previous report (in Japanese) of the results of treatment for stomach carcinoma in Japan [15], the sex ratio of patients was $66.4 \%$ men and $33.6 \%$ women, or about $2: 1$. In our study, the proportion of men was a little higher.

In this study, the proportion of patients with elevated serum CEA levels was $19.0 \%$, similar to findings $(10.6 \%$ to $50 \%$ ) in other studies $[12,14,17-25]$, and the corre- sponding proportion of patients with elevated serum CA19-9 levels was $21.8 \%$, also similar to previous findings (16.0\% to $34.6 \%$ ) [14,22-24]. These rates, however, are thought to depend on tumor progression at the time of detection. When the proportions of patients with elevated serum CEA and CA19-9 levels were assessed in accordance with the comprehensive stage grouping, it was found that the rates increased gradually with stage. At stage III or below, the positivity rates 
Table 4. Detection of independent prognostic factors by multivariate analysis with stepwise selection and Cox regression

\begin{tabular}{|c|c|c|c|c|c|c|c|}
\hline \multicolumn{2}{|c|}{ Category } & $\beta$ & $\mathrm{SE}$ & Wald & $P$ value & $\operatorname{Exp}(\beta)$ & $95 \% \mathrm{CI}$ \\
\hline \multicolumn{2}{|c|}{$\mathrm{CEA}(+)$} & 0.749 & 0.282 & 2.651 & 0.008 & 2.114 & $1.215-3.677$ \\
\hline \multirow[t]{4}{*}{ Depth } & $\mathrm{t} 1$ & & & & 0.0313 & & \\
\hline & $\mathrm{t} 2$ & 0.714 & 0.453 & 1.576 & 0.115 & 2.042 & $0.840-4.959$ \\
\hline & $\mathrm{t} 3$ & 1.216 & 0.482 & 2.524 & 0.0116 & 3.374 & $1.312-8.675$ \\
\hline & $\mathrm{t} 4$ & 1.517 & 0.772 & 1.965 & 0.0494 & 4.56 & $1.004-20.713$ \\
\hline \multirow[t]{4}{*}{$\mathrm{n}$} & 0 & & & & 0.1329 & & \\
\hline & 1 & 0.663 & 0.307 & 2.158 & 0.0309 & 1.942 & $1.063-3.547$ \\
\hline & 2 & 0.74 & 0.347 & 2.131 & 0.0331 & 2.096 & $1.061-4.138$ \\
\hline & 3 & 0.794 & 0.455 & 1.746 & 0.0808 & 2.211 & $0.907-5.389$ \\
\hline \multirow[t]{4}{*}{ ly } & 0 & & & & 0.0217 & & \\
\hline & 1 & 0.23 & 0.436 & 0.526 & 0.5987 & 1.258 & $0.535-2.959$ \\
\hline & 2 & 0.004 & 0.464 & 0.008 & 0.9939 & 1.004 & $0.404-2.494$ \\
\hline & 3 & 0.778 & 0.47 & 1.657 & 0.0031 & 2.177 & $0.867-5.465$ \\
\hline \multirow[t]{4}{*}{ ST } & 0 & & & & $<0.0001$ & & \\
\hline & 1 & 0.424 & 0.44 & 0.963 & 0.3354 & 1.527 & $0.645-3.617$ \\
\hline & 2 & 0.065 & 0.464 & 0.139 & 0.8892 & 1.067 & $0.429-2.651$ \\
\hline & 3 & 1.297 & 0.48 & 2.701 & 0.0069 & 3.66 & $1.427-9.385$ \\
\hline \multicolumn{2}{|l|}{ Age } & 0.024 & 0.009 & 2.646 & 0.0109 & 1.024 & $1.006-1.044$ \\
\hline
\end{tabular}

CI, Confidence interval; Depth, depth of invasion; $\mathrm{n}$, lymph node metastasis; ly, lymphatic invasion; ST, surgical stage

were less than $30 \%$, but the rates increased significantly, to over $50 \%$ for stage IV patients, similar to data reported previously [21].

It is likely that CEA and CA19-9 levels are increased in patients with multiple organ infiltration, advanced lymph node metastasis, peritoneal metastasis, or liver metastasis, or when other distant metastasis occurs. Therefore, we compared recurrence factors in patients with elevated and those with normal serum CEA or CA19-9 levels (Table 2). Patients with elevated serum CEA levels were at significantly higher risk of having all recurrence factors than those with normal serum CEA levels. Liver metastases, distant metastases, and lymph node metastases were selected as independent risk factors, and liver metastasis was the highest risk factor for elevated serum CEA levels. Patients with elevated serum CA19-9 levels were at significantly higher risk of having peritoneal metastases and distant metastases than those with normal serum CA19-9 levels. Distant metastases and peritoneal metastases were selected as independent risk factors, and distant metastasis was the highest risk factor for elevated serum CA19-9 levels.

In patients with gastric cancer, it has been reported that the proportion of those with elevated serum CEA levels increases with advancing cancer stage and liver metastasis [14,17-19,26]. Our study produced similar findings. One study has shown that elevated serum CA19-9 levels in gastric cancer are well correlated with various forms of metastasis [22]. No such correlation was observed in our study. Assessment of average CEA levels by comprehensive clinical stage also shows that CEA levels increase as the disease becomes more ad- vanced. Thus, we consider that the CEA level is a more useful indicator than the serum CA19-9 level for assessing the stage of gastric cancer and predicting its possible recurrence and metastasis.

We compared other clinicopathological factors in patients with primary gastric cancer who had elevated and those who had normal levels of either serum CEA or CA19-9. The proportions of patients with elevated serum CEA and CA19-9 levels were significantly higher with respect to the factors of stage grouping, depth of invasion, lymph node metastasis, lymphatic invasion, vessel invasion, and curability. Elevated serum CEA or CA19-9 levels were also correlated significantly with these factors. These findings indicate that the proportions patients with elevation of these tumor markers increases as the cancer progresses, similar to the findings of other investigators [18-25].

We also compared cumulative survival in patients with primary gastric cancer who had an elevated serum level of either CEA or CA19-9, with cumulative survival in those with a normal level of either marker. A significant difference was demonstrated between the cumulative survival curves for patients with elevated and those with normal serum CEA or CA19-9 levels (Fig. 1). Similar results in patients with gastric cancer have been reported by others [20-23].

Patients in whom both the serum CEA and CA19-9 levels were elevated had significantly worse prognoses than patients in whom the levels of both markers were normal. No significant difference was demonstrated between patients in whom the serum CEA level was elevated and the serum CA19-9 level was normal, and 
patients in whom the serum CA19-9 level was elevated and the serum CEA level was normal (Fig. 2). Similar results in patients with gastric cancer have been reported by others [23].

Comparison of cumulative survival curves according to the JCGC surgical stage grouping showed that, although there were no significant differences between patients with elevated and those with normal serum CEA or CA19-9 levels at stages I, II, and IV, the prognosis for patients at stage III who had elevated serum CEA or CA19-9 levels was worse than that for patients with normal levels (of CEA or CA19-9) $(P<0.01)$ (Fig. $3)$. Previous studies reported that the survival rate of gastric cancer patients at stages I, II, and III with serum CEA levels below $5 \mathrm{ng} / \mathrm{ml}$ was significantly better than that of patients whose serum CEA levels were above this value $[20,26]$. The survival rate of gastric cancer patients at stage I with serum CA19-9 levels below $37 \mathrm{ng} / \mathrm{ml}$ was reported to be significantly better than that of patients with levels above that value [23]. We found no significant differences between patients at stage I, II, and IV who showed elevated serum levels of either CEA or CA19-9 and those with a normal level of either marker, because of the good prognosis after curative gastrectomy at stages I and II, and the worse postoperative prognosis at stage IV.

These findings indicate that serum CEA and CA19-9 levels provide additional prognostic information in patients with primary gastric cancer. We compared cumulative survival in patients with elevated and those with normal serum levels of either CEA or CA19-9 classified for curative potential of gastrectomy according to the JCGC criteria. The gastric cancer patients with serum CEA levels below $5 \mathrm{ng} / \mathrm{ml}(\mathrm{CEA}(-)$ in Fig. 4) showed significantly better prognosis $(P<0.05)$ (Fig.4). On the other hand, no significant difference could be demonstrated between patients with elevated and those with normal serum CA19-9 levels. These findings indicate that serum CEA levels provide additional prognostic information in patients with gastric cancer who undergo gastrectomy. Similar results in patients with gastric cancer have been reported by others. One study reported that elevated serum CEA levels correlated well with other prognostic information in patients with gastric cancer who underwent curative resection of stage II and III gastric cancer [26]. We also studied cumulative survival studied in those patients who showed elevated serum CEA and CA19-9 levels before gastrectomy, in terms of measurements taken within 3 weeks after tumor resection. Patients whose serum CEA levels became normal after gastrectomy had a significantly better prognosis $(P<0.01)$ than those whose serum CEA levels remained high (Fig. 5). On the other hand, no significant difference could be demonstrated in patients with elevated serum CA19-9 levels.
These results suggest that the level of CEA could be a useful indicator of the curability of gastric resection.

To evaluate the reliability of serum CEA and CA199 levels as additional prognostic factors, we performed multivariate analysis with 14 prognostic factors, using Cox regression. In all analyses, venous invasion showed the highest value $[\exp (\beta)]$, and serum CEA levels showed higher values $[\exp (\beta)]$ than curability, lymphatic invasion, stage grouping, and depth of invasion. Serum CA19-9 levels were not a highly significant prognostic factor. To determine the independent prognostic factors, we then performed forward stepwise selection. When the cutoff level of serum CEA was increased to $10 \mathrm{ng} / \mathrm{ml}$, serum CEA, age, lymph node, metastasis, and surgical stage grouping were selected as independent prognostic factors. Previously, it was reported that preoperative CEA level was a strong prognostic factor [20], although others have reported that only serum CA19-9 level is a good prognostic factor [22]. Elevation of the serum levels of both CEA and CA19-9 was not selected as an independent prognostic factor in our study. A previous study reported that a combination assay of preoperative CEA and CA19-9 levels was an independent prognostic factor. In the present study, the lack of selection of these markers as independent prognostic factors may have been due to the small sample size. However, our data showed that serum CEA level can be an additional independent prognostic factor in patients with primary gastric cancer.

In conclusion, levels of both CEA and CA19-9 provide additional prognostic information in patients with primary gastric cancer. Furthermore, our findings indicate that the preoperative serum CEA level provides additional prognostic information and is a useful indicator of curability in patients with gastric cancer after gastrectomy, as well as being an additional independent prognostic factor in primary gastric cancer.

Acknowledgments We are grateful to Ms. H. Shouno for her assistance with data analysis.

\section{References}

1. Fuch CS, Mayer RJ. Gastric carcinoma. N Engl J Med 1995; 333:32-41.

2. Masashi F, Juei S, Toshifusa N. State of the art in the treatment of gastric cancer: from the 71st Japanese Gastric Cancer Congress. Gastric Cancer 1999;2:151-7.

3. Maruyama K. The most important prognostic factors for gastric cancer patients. A study using univariate and multivariate analyses. Scand J Gastroenterol 1987;22:63-8.

4. Yu CCW, Levison DA, Dunn JA, Ward LC, Demonakou M, Allum WH, et al. Pathological prognostic factors in the second British Stomach Cancer Group trial of adjuvant therapy in resectable gastric cancer. Br J Cancer 1995;71:1106-10.

5. Nakamura K, Ueyama T, Xuan ZX, Ambe K, Adachi Y, et al. Pathology and prognosis of gastric cancer. Findings in 10000 
patients who underwent primary gastrectomy. Cancer 1992;70: 1030-70.

6. Harrison JD, Fielding JWL. Prognostic factors for gastric cancer influencing clinical practice. World J Surg 1995;19:496-500.

7. Gold P, Freedman SO. Specific carcinoembryonic antigen of the human digestive system. J Exp Med 1965;122:467-81.

8. Holyoke ED, Chu TM, Douglass HO, Evans JT. The role of markers in the management of gastrointestinal cancer. In: Carer SK, Glastein E, Livingston RB, editors. Principles of cancer treatment. New York: McGraw-Hill; 1981. pp 456-62.

9. Wanebo H, Rao B, Pinsky C, Hoffman H. Preoperative carcinoembryonic antigen level as a prognostic indicator in colorectal cancer. N Engl J Med 1980;299:448-51.

10. Koprowski H, Steplewski Z, Mitchell K, et al. Colorectal carcinoma antigens detected by hybridoma antibodies. Somatic Cell Genet 1979;5:957-72.

11. Lowe JB, Stoolmann LM, Nair RP, et al. ELAM-1-dependent cell adhesion to vascular endothelium determined by a transfected human fucosyltransferase cDNA. Cell 1990;63:475-84.

12. Lundin J, Roberts PJ, Kuusela P, et al. The prognostic value of preoperative serum levels of CA19-9 and CEA in patients with pancreatic cancer. Br J Cancer 1994;69:515-9.

13. Sakamoto J, Watanabe T, Tokumaru T, et al. Expression of Lewis $^{\mathrm{a}}$, Lewis ${ }^{\mathrm{b}}$, Lewis ${ }^{\mathrm{y}}$, sialyl Lewis ${ }^{\mathrm{a}}$, and sialyl Lewis ${ }^{\mathrm{x}}$ blood group antigens in human gastric carcinoma and normal cancer tissue. Cancer Res 1989;49:745-52.

14. Webb A, Scott-Mackie P, Cunningham D, Norman A, Andreyev $\mathrm{J}$, O'Brien $\mathrm{M}$, et al. The prognostic value of serum and immunohistochemical tumor markers in advanced gastric cancer. Eur $\mathbf{J}$ Cancer 1996;32A:63-8.

15. Japanese Gastric Cancer Association (formerly The Japanese Research Society for Gastric Cancer and Miwa Registry Institute of Stomach Cancer). The registration report and the report of treatment results of stomach carcinoma in Japan 19701999 (in Japanese). Vol. 1-55.

16. Japanese Research Society for Gastric Cancer. Japanese classification of gastric carcinoma (First English edition). Tokyo: Kanehara; 1995.
17. Janssen $\mathrm{CW}$, Orjasater $\mathrm{H}$. Carcinoembryonic antigen in patients with gastric carcinoma. Eur J Surg Oncol 1986;12:19-23.

18. Shimizu N, Wakatsuki T, Murakami A, Yoshioka H, Hamazoe R, Kanayama $\mathrm{H}$, et al. Carcinoembryonic antigen in gastric cancer patients. Oncology 1987;44:240-4.

19. Koga T, Kano T, Souda K, Oka N, Inokuchi K. The clinical usefulness of preoperative CEA determination in gastric cancer. Jpn J Surg 1987;17:342-7.

20. Nakane Y, Okamura S, Akehira K, Baku T, Okusa T, Tanaka K, Hioki K. Correlation of preoperative carcinoembryonic antigen levels and prognosis of gastric cancer patients. Cancer 1994; 73:2703-9.

21. Maehara Y, Kusumoto T, Takahashi I, Kakeji Y, Baba H, Akazawa K, Sugimachi K. Predictive value of preoperative carcinoembryonic antigen levels for the prognosis of patients with well differentiated gastric cancer. Oncology 1994;51:234-7.

22. Kodera Y, Yamamura Y, Torii A, Uesaka K, Hirai T, Yasui K, et al. The prognostic value of preoperative serum levels of CEA and CA19-9 in patients with gastric cancer. Am J Gastroenterol 1996;91:49-53.

23. Ikeda Y, Oomori H, Koyanagi N, Mori M, Kamakura T, Minagawa $\mathrm{S}$, et al. Prognostic value of combination assays for CEA and CA19-9 in gastric cancer. Oncology 1995;52:483-6.

24. Marrelli D, Roviello F, De Stefano A, Farnetani M, Garosi L, Messano A, Pinto E. Prognostic significance of CEA, CA19-9 and CA72-4 preoperative serum levels in gastric carcinoma. Oncology 1999;57:55-62.

25. Tachibana M, Takemoto Y, Nakashima Y, Kinugasa S, Kotoh T, Dhar DK, et al. Serum carcinoembryonic antigen as a prognostic factor in resectable gastric cancer. J Am Coll Surg 1998;187:64-8.

26. Ikeda $\mathrm{Y}$, Mori M, Kajiyama K, Kamakura T, Maehara Y, Haraguchi Y, Sugimachi K. Indicative value of carcinoembryonic antigen (CEA) for liver recurrence following curative resection of stage II and III gastric cancer. Hepatogastroenterology 1996; 43:1281-7. 